\title{
RENORMALIZED SOLUTIONS FOR A NON-UNIFORMLY PARABOLIC EQUATION
}

\author{
Chao Zhang* and Shulin Zhou \\ Harbin Institute of Technology, Department of Mathematics \\ Harbin 150001, P. R. China; czhangmath@hit.edu.cn \\ Peking University, LMAM, School of Mathematical Sciences \\ Beijing 100871, P. R. China; szhou@math.pku.edu.cn
}

\begin{abstract}
In this paper we prove the existence of nonnegative renormalized solutions for the initial-boundary value problem of a non-uniformly parabolic equation. Some well-known parabolic equations are the special cases of this equation.
\end{abstract}

\section{Introduction}

Suppose that $\Omega$ is a bounded domain of $\mathbf{R}^{N}(N \geq 2)$ with Lipschitz boundary $\partial \Omega$, and $T$ is a positive number. Denote $\Omega_{T}=\Omega \times(0, T], \Sigma=\partial \Omega \times(0, T]$. In this paper we study the following non-uniformly parabolic initial-boundary value problem

$$
\begin{cases}u_{t}-\operatorname{div}\left(D_{\xi} \Phi(\nabla u)\right)=f & \text { in } \Omega_{T}, \\ u(x, t)=0 & \text { on } \Sigma, \\ u(x, 0)=u_{0}(x) & \text { on } \Omega,\end{cases}
$$

where $\Phi: \mathbf{R}^{N} \mapsto \mathbf{R}^{+}$is a $C^{1}$ nonnegative, strictly convex function, $D_{\xi} \Phi: \mathbf{R}^{N} \rightarrow \mathbf{R}$ represents the gradient of $\Phi(\xi)$ with respect to $\xi$ and $\nabla u$ represents the gradient with respect to the spatial variables $x$. Without loss of generality we may assume that $\Phi(0)=0$.

Our main assumptions are that $\Phi(\xi)$ satisfies the super-linear condition (or 1coercive condition, see [23, Chapter E])

$$
\lim _{|\xi| \rightarrow \infty} \frac{\Phi(\xi)}{|\xi|}=\infty
$$

and the symmetric condition: there exists a positive number $C>0$ such that

$$
\Phi(-\xi) \leq C \Phi(\xi), \quad \xi \in \mathbf{R}^{N}
$$

In this paper we assume

$$
u_{0} \in L^{1}(\Omega) \quad \text { and } \quad f \in L^{1}\left(\Omega_{T}\right)
$$

with

$$
u_{0} \geq 0 \text { and } f \geq 0 .
$$

There are numerous examples of $\Phi(\xi)$ satisfying structure assumptions (1.2) and (1.3). The well-known are listed as follows.

doi:10.5186/aasfm.2012.3709

2010 Mathematics Subject Classification: Primary 35D05; Secondary 35D10.

Key words: Renormalized solutions, existence, parabolic.

${ }^{*}$ Corresponding author. 
Example 1.1.

$$
\Phi(\xi)=\frac{1}{p}|\xi|^{p}, \quad p>1 .
$$

In this case, equation (1.1) is the parabolic counterpart of the $p$-Laplacian.

Example 1.2.

$$
\Phi(\xi)=\frac{1}{p_{1}}\left|\xi_{1}\right|^{p_{1}}+\frac{1}{p_{2}}\left|\xi_{2}\right|^{p_{2}}+\cdots+\frac{1}{p_{N}}\left|\xi_{N}\right|^{p_{N}}, \quad p_{i}>1, i=1,2, \ldots, N,
$$

where $\xi=\left(\xi_{1}, \xi_{2}, \ldots, \xi_{N}\right)$. In this case, equation (1.1) is the parabolic counterpart of the anisotropic $p$-Laplacian. (See [25, Chapter 2].)

Example 1.3.

$$
\Phi(\xi)=|\xi| \log (1+|\xi|) .
$$

(See [12, Chapter 4] and [20].)

Example 1.4.

$$
\Phi(\xi)=|\xi| L_{k}(|\xi|),
$$

where $L_{i}(s)=\log \left(1+L_{i-1}(s)\right)(i=1,2, \ldots, k)$ and $L_{0}(s)=\log (1+s)$ for $s \geq 0$. (See [22].)

Example 1.5.

$$
\Phi(\xi)=e^{\frac{|\xi|^{2}}{2}}-1
$$

(See [17], [24] and [28].)

Recently, Cai and Zhou [10] considered problem (1.1) without the right hand side $f$ under structure assumptions (1.2), (1.3) and integrability condition $u_{0} \in L^{2}(\Omega)$ and proved the existence and uniqueness of weak solutions. This paper is a continuation of [10]. We are interested in the study of the well-posedness of problem (1.1) with $L^{1}$ data. The existence techniques akin to the techniques of this paper were used in the elliptic case by Boccardo and Gallouët in [7] and [8]. The first paper [7] also contained parabolic results, further developed in [6]. However, the counterexamples by Serrin, [32], indicated that with nontrivial right hand side uniqueness might fail when $n \geq 3$ with the usual definition. Thus some extra conditions on the distributional solutions were needed in order to ensure both existence and uniqueness. The three different definitions for this purpose were independently introduced by Bénilan et al. [2] (entropy solutions), by Dall'Aglio [16] (SOLA, Solutions Obtained as Limit of Approximations) and by Lions and Murat [27] (renormalized solutions, see also [20]). In the parabolic case, one should consult [16] (SOLA), [3] (renormalized solutions) and [29] (entropy solutions).

One result on this topic can be found in [34] where via the introduction of the notion of entropy solutions. We proved that there exists an entropy solution for problem (1.1) under assumptions (1.2), (1.3) and (1.4). Besides, in [33] we studied the following nonlinear parabolic problem

$$
\begin{cases}u_{t}-\operatorname{div}\left(|\nabla u|^{p(x)-2} \nabla u\right)=f & \text { in } \Omega_{T}, \\ u=0 & \text { on } \Sigma, \\ u(x, 0)=u_{0}(x) & \text { on } \Omega,\end{cases}
$$


where the variable exponent $p: \bar{\Omega} \rightarrow(1,+\infty)$ is a continuous function, $f \in L^{1}\left(\Omega_{T}\right)$ and $u_{0} \in L^{1}(\Omega)$. We proved the existence and uniqueness of both renormalized solutions and entropy solutions for problem (1.6) and discovered the equivalence of renormalized solutions and entropy solutions. This motivates us to study problem (1.1) in the framework of renormalized solutions. The notion of renormalized solutions was first introduced by DiPerna and Lions [20] for the study of Boltamann equation. It was then adapted to the study of some nonlinear elliptic and parabolic problems and evolution problems in fluid mechanics $([3,4,5,9,15,26])$. We hope that the renormalized solution is still existent and unique, and it is equivalent to the entropy solution of problem (1.1). However, so far we can only get the existence of renormalized solutions for problem (1.1) under (1.2), (1.3), (1.4) and additional (1.5). The uniqueness of renormalized solutions and the equivalence of renormalized solutions and entropy solutions remain open. The main difficulty lies on that there is no growth condition for function $\Phi(\xi)$. To our knowledge, growth conditions such as polynomial growth or exponential growth for function $\Phi(\xi)$ played an extremely important role, for example in $[1,11,24]$, when parabolic problems or variational problems related to (1.1) were studied. If some condition such as $\Delta_{2}$-condition, i.e., there exists a positive constant $K$ such that for every $\xi>0$ such that

$$
\Phi(2 \xi) \leq K \Phi(\xi)
$$

is further assumed on function $\Phi(\xi)$, it is possible to prove the uniqueness result of the renormalized solutions, and the equivalence of renormalized solutions and entropy solutions for problem (1.1) without the nonnegativity assumption (1.5).

Let $T_{k}$ denote the truncation function at height $k \geq 0$ :

$$
T_{k}(r)=\min \{k, \max \{r,-k\}\}= \begin{cases}k & \text { if } r \geq k, \\ r & \text { if }|r|<k, \\ -k & \text { if } r \leq-k,\end{cases}
$$

and its primitive $\Theta_{k}: \mathbf{R} \rightarrow \mathbf{R}^{+}$by

$$
\Theta_{k}(r)=\int_{0}^{r} T_{k}(s) d s= \begin{cases}\frac{r^{2}}{2} & \text { if }|r| \leq k, \\ k|r|-\frac{k^{2}}{2} & \text { if }|r| \geq k .\end{cases}
$$

It is obvious that $\Theta_{k}(r) \geq 0$ and $\Theta_{k}(r) \leq k|r|$.

Next we define the very weak gradient of a measurable function $u$ with $T_{k}(u) \in$ $L^{1}\left(0, T ; W_{0}^{1,1}(\Omega)\right)$. As a matter of the fact, working as in Lemma 2.1 of [2] we can prove the following result:

Proposition 1.6. For every measurable function $u$ on $\Omega_{T}$ such that $T_{k}(u)$ belongs to $L^{1}\left(0, T ; W_{0}^{1,1}(\Omega)\right)$ for every $k>0$, there exists a unique measurable function $v: \Omega_{T} \rightarrow \mathbf{R}^{N}$, such that

$$
\nabla T_{k}(u)=v \chi_{\{|u|<k\}} \quad \text { almost everywhere in } \Omega_{T} \text { and for every } k>0,
$$

where $\chi_{E}$ denotes the characteristic function of a measurable set $E$. Moreover, if $u$ belongs to $L^{1}\left(0, T ; W_{0}^{1,1}(\Omega)\right)$, then $v$ coincides with the weak gradient of $u$.

From Proposition 1.6, we denote $v=\nabla u$, which is called the very weak gradient of $u$. The notion of the very weak gradient allows us to give the following definition of renormalized solutions for problem (1.1). Denote $z=(x, t), d z=d x d t$. 
Definition 1.7. A nonnegative function $u \in C\left([0, T] ; L^{1}(\Omega)\right)$ such that $T_{k}(u) \in$ $L^{1}\left(0, T ; W_{0}^{1,1}(\Omega)\right)$ is called a renormalized solution to problem (1.1) if the following conditions are satisfied:

(i) $\lim _{n \rightarrow \infty} \int_{\left\{(x, t) \in \Omega_{T}: n \leq|u(x, t)| \leq n+1\right\}} D_{\xi} \Phi(\nabla u) \cdot \nabla u d z=0$;

(ii) For every nonnegative function $\varphi \in C^{1}\left(\bar{\Omega}_{T}\right)$ with $\varphi(\cdot, T)=0$ and $S \in$ $C^{\infty}\left(\mathbf{R}^{+}\right)$satisfying that $S^{\prime}$ has a compact support and $S^{\prime \prime}$ is non-positive,

$$
\begin{aligned}
& -\int_{\Omega} S\left(u_{0}\right) \varphi(x, 0) d x-\int_{0}^{T} \int_{\Omega} S(u) \frac{\partial \varphi}{\partial t} d z \\
& \quad+\int_{0}^{T} \int_{\Omega}\left[S^{\prime}(u) D_{\xi} \Phi(\nabla u) \cdot \nabla \varphi+S^{\prime \prime}(u) D_{\xi} \Phi(\nabla u) \cdot \nabla u \varphi\right] d z \\
& \geq \int_{0}^{T} \int_{\Omega} f S^{\prime}(u) \varphi d z
\end{aligned}
$$

holds.

Now we state our main result.

Theorem 1.8. Under structure assumptions (1.2), (1.3), integrability condition (1.4) and nonnegativity condition (1.5), there exists a renormalized solution for problem (1.1).

The rest of this paper is organized as follows. In Section 2, we state some basic results that will be used later. We will prove the main result in Section 3. In the following sections $C$ will represent a generic constant that may change from line to line even if in the same inequality.

\section{Preliminaries}

Let $\Phi(\xi)$ be a nonnegative convex function. We define the polar function of $\Phi(\xi)$ as

$$
\Psi(\eta)=\sup _{\xi \in \mathbf{R}^{N}}\{\eta \cdot \xi-\Phi(\xi)\}
$$

which is also known as the Legendre transform of $\Phi(\xi)$. It is obvious that $\Psi(\eta)$ is a convex function. In the following we will list several lemmas.

Definition 2.1. [23, Definition 4.1.3] Let $C \subset \mathbf{R}^{N}$ be convex. The mapping $F: C \rightarrow \mathbf{R}^{N}$ is said to be monotone [resp. strictly monotone] on $\mathrm{C}$ when, for all $x$ and $x^{\prime}$ in $C$,

$$
\begin{aligned}
\left\langle F(x)-F\left(x^{\prime}\right), x-x^{\prime}\right\rangle & \geq 0, \\
{\left[\operatorname{resp} .\left\langle F(x)-F\left(x^{\prime}\right), x-x^{\prime}\right\rangle\right.} & \left.>0 \quad \text { whenever } \quad x \neq x^{\prime}\right] .
\end{aligned}
$$

Lemma 2.2. [23, Theorem 4.1.4] Let $f$ be a function differentiable on an open set $\Omega \subset \mathbf{R}^{N}$ and let $C$ be a convex subset of $\Omega$. Then, $f$ is convex [resp. strictly convex] on $C$ if and only if its gradient $\nabla f$ is monotone [resp. strictly monotone] on $C$. 
Lemma 2.3. Suppose that $\Phi(\xi)$ is a convex $C^{1}$ function with $\Phi(0)=0$. Then we have for all $\xi, \zeta \in \mathbf{R}^{N}$,

$$
\begin{aligned}
\Phi(\xi) & \leq \xi \cdot D \Phi(\xi), \\
(D \Phi(\xi)-D \Phi(\zeta)) \cdot(\xi-\zeta) & \geq 0 .
\end{aligned}
$$

Lemma 2.4. [10, Lemma 2.2] Suppose that $\Phi(\xi)$ is a nonnegative convex $C^{1}$ function and $\Psi(\eta)$ is its polar function. Then we have for $\xi, \eta, \zeta \in \mathbf{R}^{N}$,

$$
\begin{aligned}
\xi \cdot \eta & \leq \Phi(\xi)+\Psi(\eta), \\
\Psi(D \Phi(\zeta))+\Phi(\zeta) & =D \Phi(\zeta) \cdot \zeta .
\end{aligned}
$$

Lemma 2.5. [21, Chapter 3] Suppose that $\Phi(\xi)$ is a nonnegative convex function with $\Phi(0)=0$, which satisfies (1.2). Then its polar function $\Psi(\eta)$ in (2.1) is a welldefined, nonnegative function in $\mathbf{R}^{N}$, which also satisfies (1.2).

Lemma 2.6. [30, Chapter 4] Let $D \subset \mathbf{R}^{N}$ be measurable with finite Lebesgue measure and $f_{k} \in L^{1}(D)$ and $g_{k} \in L^{1}(D)(k=1,2, \ldots)$, and

$$
\left|f_{k}(x)\right| \leq g_{k}(x) \text { a.e. } x \in D, k=1,2, \ldots
$$

If

$$
\lim _{k \rightarrow \infty} f_{k}(x)=f(x), \quad \lim _{k \rightarrow \infty} g_{k}(x)=g(x) \text { a.e. } x \in D
$$

and

$$
\lim _{k \rightarrow \infty} \int_{D} g_{k}(x) d x=\int_{D} g(x) d x<+\infty
$$

then we have

$$
\lim _{k \rightarrow \infty} \int_{D} f_{k}(x) d x=\int_{D} f(x) d x .
$$

Lemma 2.7. [19, Proposition 9.1c] or [34, Lemma 2.7] Let $D \subset \mathbf{R}^{N}$ be measurable with finite Lebesgue measure, and let $\left\{f_{n}\right\}$ be a sequence of functions in $L^{p}(D)(p \geq 1)$ such that

$$
\begin{array}{ll}
f_{n} \rightarrow f & \text { weakly in } L^{p}(D), \\
f_{n} \rightarrow g & \text { a.e. in } D .
\end{array}
$$

Then $f=g$ a.e. in $D$.

Lemma 2.8. Let $D \subset \mathbf{R}^{N}$ be measurable with finite Lebesgue measure. Suppose that $\left\{a_{n}(x)\right\} \subset L^{\infty}(D)$ and $\left\{g_{n}(x)\right\} \subset L^{1}(D)$ are two sequences such that

$$
a_{n} \rightarrow a \quad \text { a.e. in } D \quad \text { and } \quad a_{n} \rightarrow a \quad \text { weakly-* in } L^{\infty}(D)
$$

and

$$
g_{n} \rightarrow g \quad \text { weakly in } L^{1}(D)
$$

Then

$$
a_{n} g_{n} \rightarrow a g \quad \text { weakly in } L^{1}(D) .
$$

Proof. By assumptions, we can know that there exist two positive numbers $M_{1}, M_{2}$ such that

$$
\left\|a_{n}\right\|_{L^{\infty}(D)},\|a\|_{L^{\infty}(D)} \leq M_{1}, \quad\left\|g_{n}\right\|_{L^{1}(D)},\|g\|_{L^{1}(D)} \leq M_{2} .
$$


According to Egorov theorem, for every $\delta>0$, there exists a measurable set $E_{\delta} \subset D$ such that $\left|D \backslash E_{\delta}\right| \leq \delta$ and

$$
a_{n} \rightarrow a \quad \text { uniformly in } E_{\delta} .
$$

Then for every $\varepsilon>0$, there exists $N_{1}>0$ such that when $n>N_{1}$,

$$
\sup _{E_{\delta}}\left|a_{n}-a\right|<\frac{\varepsilon}{3 M_{2} M_{3}},
$$

where $M_{3}$ is a positive number determined later.

For fixed $\psi \in L^{\infty}(D)$, we can write

$$
\int_{D}\left(a_{n} g_{n}-a g\right) \psi d x=\int_{D}\left(a_{n} g_{n}-a g_{n}\right) \psi d x+\int_{D}\left(g_{n}-g\right) a \psi d x:=I_{1}+I_{2} .
$$

Since $g_{n} \rightarrow g$ weakly in $L^{1}(D)$ and $a \psi \in L^{\infty}(D)$, it is easy to see that there exists $N_{2}>0$ such that

when $n>N_{2}$.

$$
\left|I_{2}\right|<\frac{\varepsilon}{3}
$$

Next we estimate $I_{1}$ as follows. Set

$$
\|\psi\|_{L^{\infty}(D)} \leq M_{3}
$$

For all $n>N_{0}=\max \left\{N_{1}, N_{2}\right\}$, we have

$$
\begin{aligned}
I_{1} & \leq \int_{D}\left|\left(a_{n} g_{n}-a g_{n}\right) \psi\right| d x=\int_{E_{\delta}}\left|\left(a_{n}-a\right) g_{n} \psi\right| d x+\int_{D \backslash E_{\delta}}\left|\left(a_{n}-a\right) g_{n} \psi\right| d x \\
& \leq \sup _{E_{\delta}}\left|a_{n}-a\right| \cdot\|\psi\|_{L^{\infty}(D)}\left\|g_{n}\right\|_{L^{1}(D)}+\left(\left\|a_{n}\right\|_{L^{\infty}(D)}+\|a\|_{L^{\infty}(D)}\right)\|\psi\|_{L^{\infty}(D)} \int_{D \backslash E_{\delta}}\left|g_{n}\right| d x \\
& \leq \frac{\varepsilon}{3}+2 M_{1} M_{3} \int_{D \backslash E_{\delta}}\left|g_{n}\right| d x .
\end{aligned}
$$

We recall that Dunford-Pettis theorem ensures that a sequence in $L^{1}(D)$ is weakly convergent in $L^{1}(D)$ if and only if it is equi-integrable. Thus by Dunford-Pettis theorem, for $\varepsilon_{0}=\varepsilon /\left(6 M_{1} M_{3}\right)$, there exists a positive number $\delta_{0}=\delta_{0}(\varepsilon)$ such that

$$
\int_{A}\left|g_{n}\right| d x<\varepsilon_{0}
$$

for every subset $A \subset D$ with $|A|<\delta_{0}$. Choosing $\delta=\delta_{0}$ it follows that $\left|D \backslash E_{\delta}\right|<\delta_{0}$ and

$$
2 M_{1} M_{3} \int_{D \backslash E_{\delta}}\left|g_{n}\right| d x<\frac{\varepsilon}{3} .
$$

Therefore, combining the above estimates, we obtain

$$
\left|I_{1}\right|<\frac{2 \varepsilon}{3}
$$

Hence, we conclude that

$$
\left|\int_{D}\left(a_{n} g_{n}-a g\right) \psi d x\right|<\varepsilon \quad \text { for all } n>N_{0}
$$

which implies that

$$
\lim _{n \rightarrow \infty} \int_{D}\left(a_{n} g_{n}-a g\right) \psi d x=0 \quad \text { for all } \psi \in L^{\infty}(D) .
$$


This finishes the proof.

Lemma 2.9. [14, Chapter 3] and [31] Suppose that $\Phi(\xi)$ is a nonnegative convex function satisfying (1.2). Let $D \subset \mathbf{R}^{N}$ be a measurable with finite Lebesgue measure $|D|$ and let $\left\{f_{k}\right\} \subset L^{1}\left(D ; \mathbf{R}^{N}\right)$ be a sequence satisfying that

$$
\int_{D} \Phi\left(f_{k}\right) d x \leq C
$$

where $C$ is a positive constant. Then there exist a subsequence $\left\{f_{k_{j}}\right\} \subset\left\{f_{k}\right\}$ and a function $f \in L^{1}\left(D ; \mathbf{R}^{N}\right)$ such that

$$
f_{k_{j}} \rightarrow f \quad \text { weakly in } L^{1}\left(D ; \mathbf{R}^{N}\right) \text { as } j \rightarrow \infty
$$

and

$$
\int_{D} \Phi(f) d x \leq \liminf _{j \rightarrow \infty} \int_{D} \Phi\left(f_{k_{j}}\right) d x \leq C .
$$

For the convenience of the readers, let us recall the definition of weak solutions for problem (1.1) and the main results in [10].

Definition 2.10. A function $u: \bar{\Omega} \times[0, T] \rightarrow \mathbf{R}$ is a weak solution of problem (1.1) if the following conditions are satisfied:

(i) $u \in C\left([0, T] ; L^{2}(\Omega)\right) \cap L^{1}\left(0, T ; W_{0}^{1,1}(\Omega)\right)$ with

$$
\int_{\Omega_{T}} D_{\xi} \Phi(\nabla u) \cdot \nabla u d z<\infty
$$

(ii) For any $\varphi \in C^{1}\left(\bar{\Omega}_{T}\right)$ with $\varphi(\cdot, T)=0$ and $\left.\varphi(\cdot, t)\right|_{\partial \Omega}=0$, we have

$$
-\int_{\Omega} u_{0}(x) \varphi(x, 0) d x+\int_{\Omega_{T}}\left[-u \varphi_{t}+D_{\xi} \Phi(\nabla u) \cdot \nabla \varphi\right] d z=\int_{\Omega_{T}} f \varphi d z .
$$

Lemma 2.11. [10, Theorem 1.2] Let the structure assumptions (1.2) and (1.3) be satisfied. If $u_{0} \in L^{2}(\Omega)$ and $f=0$, then there exists a unique weak solution for the initial-boundary value problem (1.1).

Corollary 2.12. If we assume $f \in L^{2}\left(\Omega_{T}\right)$ the existence and uniqueness of weak solutions of problem (1.1) can be obtained working as in the proof of Lemma 2.11.

Remark 2.13. Let $u$ be a weak solution in Definition 2.10. By using the approximation technique (see [13, Chapter 3] or [18, Chapter 2]) we have for every $\varphi \in C^{1}\left(\bar{\Omega}_{T}\right)$ with $\left.\varphi(\cdot, t)\right|_{\partial \Omega}=0$, each $t \in[0, T]$,

$$
\left.\int_{\Omega} u \varphi d x\right|_{0} ^{t}+\int_{0}^{t} \int_{\Omega}\left[-u \varphi_{t}+D_{\xi} \Phi(\nabla u) \cdot \nabla \varphi\right] d x d \tau=\int_{0}^{t} \int_{\Omega} f \varphi d x d \tau .
$$

Remark 2.14. Let $u$ be a weak solution in Definition 2.10 with $f=0$. We can formally choose $u$ as a test function in (2.10) to obtain an energy type estimate. That is, for a.e. $t \in[0, T]$,

$$
\frac{1}{2}\|u(t)\|_{L^{2}(\Omega)}^{2}+\int_{0}^{t} \int_{\Omega} D_{\xi} \Phi(\nabla u) \cdot \nabla u d x d \tau=\frac{1}{2}\left\|u_{0}\right\|_{L^{2}(\Omega)}^{2} .
$$




\section{The proof of main result}

Now we are ready to prove our main result: the existence of renormalized solutions of problem (1.1). Our proof follows rather standard (but nontrivial) procedure. First $f$ and $u_{0}$ are estimated by smooth functions for which the existence of weak solutions is known. Then, by using the definition of a weak solution, we show that the sequence of solutions forms a Cauchy sequence in a suitable norm (passing to a subsequence if necessary). However, this is not enough to pass to a limit under the integral sign but more information is needed on the gradients. The next step is to show by a compactness argument that the gradients converge almost everywhere. This can be done without the growth bounds for function $\Phi$. The final step is to validate that the limit is a renormalized solution. Some of the reasoning is based on the ideas developed in [2], [7], [8] and [29].

Proof of Theorem 1.8. We first introduce the approximate problems. Let $\left\{f_{n}\right\} \subset C_{0}^{\infty}\left(\Omega_{T}\right)$ and $\left\{u_{0 n}\right\} \subset C_{0}^{\infty}(\Omega)$ be two nonnegative sequences of functions strongly convergent respectively to $f$ in $L^{1}\left(\Omega_{T}\right)$ and to $u_{0}$ in $L^{1}(\Omega)$ with $f, u_{0} \geq 0$, such that

$$
\left\|f_{n}\right\|_{L^{1}\left(\Omega_{T}\right)} \leq\|f\|_{L^{1}\left(\Omega_{T}\right)},\left\|u_{0 n}\right\|_{L^{1}(\Omega)} \leq\left\|u_{0}\right\|_{L^{1}(\Omega)} .
$$

Let us consider the approximate problems

$$
\begin{cases}\left(u_{n}\right)_{t}-\operatorname{div}\left(D_{\xi} \Phi\left(\nabla u_{n}\right)\right)=f_{n} & \text { in } \Omega_{T} \\ u_{n}=0 & \text { on } \Sigma \\ u_{n}(x, 0)=u_{0 n} & \text { on } \Omega .\end{cases}
$$

By virtue of Lemma 2.11 (see also Corollary 2.12) we can find nonnegative $u_{n} \in$ $C\left([0, T] ; L^{2}(\Omega)\right) \cap L^{1}\left(0, T ; W_{0}^{1,1}(\Omega)\right)$, that is a weak solution of problem (3.2) in the sense of Definition 2.10. It follows from $f_{n} \in C_{0}^{\infty}\left(\Omega_{T}\right) \subset L^{2}\left(\Omega_{T}\right), D_{\xi} \Phi\left(\nabla u_{n}\right) \in$ $L^{1}\left(\Omega_{T}\right)$ (see [10] for details) and the equation (3.2) that $\left(u_{n}\right)_{t} \in L^{1}\left(0, T ; W^{-1,1}(\Omega)\right)+$ $L^{2}\left(\Omega_{T}\right)$ and

$$
\int_{\Omega_{T}} D_{\xi} \Phi\left(\nabla u_{n}\right) \cdot \nabla u_{n} d z<\infty
$$

Our aim is to prove that a subsequence of these approximate solutions $\left\{u_{n}\right\}$ converges to a nonnegative measurable function $u$, which is an renormalized solution of problem (1.1). We will divide the proof into several steps. Although some of the arguments are not new, we present a self-contained proof for the sake of clarity and readability.

Using an approximation argument as in Remark 2.13 and Remark 2.14, we can choose $T_{k}\left(u_{n}\right) \chi_{(0, t)}$ as a test function in (3.2) to have

$$
\begin{aligned}
& \int_{\Omega} \Theta_{k}\left(u_{n}\right)(t) d x-\int_{\Omega} \Theta_{k}\left(u_{0 n}\right) d x+\int_{0}^{t} \int_{\Omega} D_{\xi} \Phi\left(\nabla T_{k}\left(u_{n}\right)\right) \cdot \nabla T_{k}\left(u_{n}\right) d x d s \\
& =\int_{0}^{t} \int_{\Omega} f_{n} T_{k}\left(u_{n}\right) d x d s .
\end{aligned}
$$

It follows from the definition (1.7) of $\Theta_{k}(r)$ and (3.1) that

$$
\begin{aligned}
& \int_{0}^{t} \int_{\Omega} D_{\xi} \Phi\left(\nabla T_{k}\left(u_{n}\right)\right) \cdot \nabla T_{k}\left(u_{n}\right) d x d s+\int_{\Omega} \Theta_{k}\left(u_{n}\right)(t) d x \\
& \leq k\left(\left\|f_{n}\right\|_{L^{1}\left(\Omega_{T}\right)}+\left\|u_{0 n}\right\|_{L^{1}(\Omega)}\right) \leq k\left(\|f\|_{L^{1}\left(\Omega_{T}\right)}+\left\|u_{0}\right\|_{L^{1}(\Omega)}\right) .
\end{aligned}
$$


Recalling (2.2), we have

$$
\int_{\Omega_{T}} \Phi\left(\nabla T_{k}\left(u_{n}\right)\right) d z \leq \int_{\Omega_{T}} D_{\xi} \Phi\left(T_{k}\left(\nabla u_{n}\right)\right) \cdot \nabla T_{k}\left(u_{n}\right) d z \leq C k,
$$

which implies from (1.2) that

$$
\int_{\Omega_{T}}\left|\nabla T_{k}\left(u_{n}\right)\right| d z \leq C(k+1)
$$

that is $T_{k}\left(u_{n}\right)$ is bounded in $L^{1}\left(0, T ; W_{0}^{1,1}(\Omega)\right)$.

If we choose $k=1$ in the inequality (3.5), then for a.e. $t \in[0, T]$,

$$
\int_{\Omega} \Theta_{1}\left(u_{n}(t)\right) d x \leq\|f\|_{L^{1}\left(\Omega_{T}\right)}+\left\|u_{0}\right\|_{L^{1}(\Omega)} .
$$

Moreover,

$$
\int_{\Omega}\left|u_{n}(t)\right| d x \leq \operatorname{meas}(\Omega)+\|f\|_{L^{1}\left(\Omega_{T}\right)}+\left\|u_{0}\right\|_{L^{1}(\Omega)} .
$$

Thus we obtain

$$
\left\|u_{n}\right\|_{L^{\infty}\left(0, T ; L^{1}(\Omega)\right)} \leq C .
$$

Step 1. We shall prove that $\left\{u_{n}\right\}$ converges in $C\left([0, T] ; L^{1}(\Omega)\right)$ and we shall find a subsequence which is almost everywhere convergent in $\Omega_{T}$.

Let $m$ and $n$ be two integers, then from (3.2) we can write the weak form as

$$
\begin{aligned}
& \int_{0}^{T}\left\langle\left(u_{n}-u_{m}\right)_{t}, \phi\right\rangle d t+\int_{\Omega_{T}}\left[D_{\xi} \Phi\left(\nabla u_{n}\right)-D_{\xi} \Phi\left(\nabla u_{m}\right)\right] \cdot \nabla \phi d z \\
& =\int_{\Omega_{T}}\left(f_{n}-f_{m}\right) \phi d z,
\end{aligned}
$$

for all $\phi \in C_{0}^{1}\left(\bar{\Omega}_{T}\right)$. Recalling (2.4), (2.5), (1.3) and (3.3), we observe that

$$
\begin{aligned}
\left|D_{\xi} \Phi\left(\nabla u_{n}\right) \cdot \nabla u_{m}\right| & \leq \Phi\left(\nabla u_{m}\right)+\Phi\left(-\nabla u_{m}\right)+\Psi\left(D_{\xi} \Phi\left(\nabla u_{n}\right)\right) \\
& \leq(C+1) \Phi\left(\nabla u_{m}\right)+D_{\xi} \Phi\left(\nabla u_{n}\right) \cdot \nabla u_{n} \\
& \leq(C+1) D_{\xi} \Phi\left(\nabla u_{m}\right) \cdot \nabla u_{m}+D_{\xi} \Phi\left(\nabla u_{n}\right) \cdot \nabla u_{n} \in L^{1}\left(\Omega_{T}\right) .
\end{aligned}
$$

Denote

$$
\alpha_{n, m}=\int_{\Omega_{T}}\left|f_{n}-f_{m}\right| d z+\int_{\Omega}\left|u_{0 n}-u_{0 m}\right| d x .
$$

We know that

$$
\lim _{n, m \rightarrow \infty} \alpha_{n, m}=0 .
$$

Using an approximation argument as above, we conclude that $w=T_{1}\left(u_{n}-u_{m}\right) \chi_{(0, t)}$ with $t \leq T$ can be a test function in (3.9). From (2.3), discarding the positive term we get

$$
\begin{aligned}
\int_{\Omega} \Theta_{1}\left(u_{n}-u_{m}\right)(t) d x & \leq \int_{\Omega} \Theta_{1}\left(u_{0 n}-u_{0 m}\right) d x+\left\|f_{n}-f_{m}\right\|_{L^{1}\left(\Omega_{T}\right)} \\
& \leq\left\|u_{0 n}-u_{0 m}\right\|_{L^{1}(\Omega)}+\left\|f_{n}-f_{m}\right\|_{L^{1}\left(\Omega_{T}\right)}=\alpha_{n, m}
\end{aligned}
$$


Therefore, we conclude from the definition (1.7) of $\Theta_{k}(r)$ that

$$
\begin{aligned}
& \int_{\left\{\left|u_{n}-u_{m}\right|<1\right\}} \frac{\left|u_{n}-u_{m}\right|^{2}(t)}{2} d x+\int_{\left\{\left|u_{n}-u_{m}\right| \geq 1\right\}} \frac{\left|u_{n}-u_{m}\right|(t)}{2} d x \\
& \leq \int_{\Omega}\left[\Theta_{1}\left(u_{n}-u_{m}\right)\right](t) d x \leq \alpha_{n, m} .
\end{aligned}
$$

It follows from Hölder's inequality that

$$
\begin{aligned}
\int_{\Omega}\left|u_{n}-u_{m}\right|(t) d x & =\int_{\left\{\left|u_{n}-u_{m}\right|<1\right\}}\left|u_{n}-u_{m}\right|(t) d x+\int_{\left\{\left|u_{n}-u_{m}\right| \geq 1\right\}}\left|u_{n}-u_{m}\right|(t) d x \\
& \leq\left(\int_{\left\{\left|u_{n}-u_{m}\right|<1\right\}}\left|u_{n}-u_{m}\right|^{2}(t) d x\right)^{\frac{1}{2}} \operatorname{meas}(\Omega)^{\frac{1}{2}}+2 \alpha_{n, m} \\
& \leq(2 \operatorname{meas}(\Omega))^{\frac{1}{2}} \alpha_{n, m}^{\frac{1}{2}}+2 \alpha_{n, m} .
\end{aligned}
$$

Thus we get

$$
\left\|u_{n}-u_{m}\right\|_{C\left([0, T] ; L^{1}(\Omega)\right)} \rightarrow 0 \quad \text { as } n, m \rightarrow \infty
$$

i.e., $\left\{u_{n}\right\}$ is a Cauchy sequence in $C\left([0, T] ; L^{1}(\Omega)\right)$. Then $u_{n}$ converges to $u$ in $C\left([0, T] ; L^{1}(\Omega)\right)$. We find an a.e. convergent subsequence (still denoted by $\left\{u_{n}\right\}$ ) in $\Omega_{T}$ such that

$$
u_{n} \rightarrow u \quad \text { a.e. in } \Omega_{T} .
$$

Recalling (3.6) and Lemma 2.9, we may draw a subsequence (we also denote it by the original sequence for simplicity) such that

$$
\nabla T_{k}\left(u_{n}\right) \rightarrow \eta_{k} \quad \text { weakly in } L^{1}\left(\Omega_{T}\right)
$$

and

$$
\int_{\Omega_{T}} \Phi\left(\eta_{k}\right) d z \leq C k
$$

In view of (3.11), we conclude that $\eta_{k}=\nabla T_{k}(u)$ a.e. in $\Omega_{T}$.

Step 2. We shall prove that the sequence $\left\{\nabla u_{n}\right\}$ converges almost everywhere in $\Omega_{T}$ to $\nabla u$ (up to a subsequence).

We first claim that $\left\{\nabla u_{n}\right\}$ is a Cauchy sequence in measure. Let $\delta>0$, and denote

$$
\begin{aligned}
& E_{1}:=\left\{(x, t) \in \Omega_{T}:\left|\nabla u_{n}\right|>h\right\} \cup\left\{(x, t) \in \Omega_{T}:\left|\nabla u_{m}\right|>h\right\} \\
& E_{2}:=\left\{(x, t) \in \Omega_{T}:\left|u_{n}-u_{m}\right|>1\right\}
\end{aligned}
$$

and

$$
E_{3}:=\left\{(x, t) \in \Omega_{T}:\left|\nabla u_{n}\right| \leq h,\left|\nabla u_{m}\right| \leq h,\left|u_{n}-u_{m}\right| \leq 1,\left|\nabla u_{n}-\nabla u_{m}\right|>\delta\right\},
$$

where $h$ will be chosen later. It is obvious that

$$
\left\{(x, t) \in \Omega_{T}:\left|\nabla u_{n}-\nabla u_{m}\right|>\delta\right\} \subset E_{1} \cup E_{2} \cup E_{3} .
$$

For $k \geq 0$, we can write $\left\{(x, t) \in \Omega_{T}:\left|\nabla u_{n}\right| \geq h\right\} \subset\left\{(x, t) \in \Omega_{T}:\left|u_{n}\right| \geq k\right\} \cup\left\{(x, t) \in \Omega_{T}:\left|\nabla T_{k}\left(u_{n}\right)\right| \geq h\right\}$. Thus, applying (3.8), (1.2) and (3.7), there exist constants $C>0$ such that

$$
\operatorname{meas}\left\{(x, t) \in \Omega_{T}:\left|\nabla u_{n}\right| \geq h\right\} \leq \frac{C}{k}+\frac{C k}{h},
$$


when $h$ is large appropriately. By choosing $k=C h^{\frac{1}{2}}$, we deduce that

$$
\operatorname{meas}\left\{(x, t) \in \Omega_{T}:\left|\nabla u_{n}\right| \geq h\right\} \leq C h^{-\frac{1}{2}} .
$$

Let $\varepsilon>0$. We may let $h=h(\varepsilon)$ large enough such that

$$
\operatorname{meas}\left(E_{1}\right) \leq \varepsilon / 3 \text { for all } n, m \geq 0 \text {. }
$$

On the other hand, by Step 1, we know that $\left\{u_{n}\right\}$ is a Cauchy sequence in $L^{1}\left(\Omega_{T}\right)$. Then there exists $N_{1}(\varepsilon) \in \mathbf{N}$ such that

$$
\operatorname{meas}\left(E_{2}\right) \leq \varepsilon / 3 \text { for all } n, m \geq N_{1}(\varepsilon) .
$$

Moreover, since $\Phi$ is $C^{1}$ and strictly convex, then from Lemma 2.2 and Definition 2.1, there exists a real valued function $m(h, \delta)>0$ such that

$$
(D \Phi(\xi)-D \Phi(\zeta)) \cdot(\xi-\zeta) \geq m(h, \delta)>0
$$

for all $\xi, \zeta \in \mathbf{R}^{N}$ with $|\xi|,|\zeta| \leq h,|\xi-\zeta| \geq \delta$. By taking $T_{1}\left(u_{n}-u_{m}\right)$ as a test function in (3.9), we obtain

$$
\begin{aligned}
m(h, \delta) \operatorname{meas}\left(E_{3}\right) & \leq \int_{E_{3}}\left[D_{\xi} \Phi\left(\nabla u_{n}\right)-D_{\xi} \Phi\left(\nabla u_{m}\right)\right] \cdot\left(\nabla u_{n}-\nabla u_{m}\right) d z \\
& \leq \int_{\Omega_{T}}\left[D_{\xi} \Phi\left(\nabla u_{n}\right)-D_{\xi} \Phi\left(\nabla u_{m}\right)\right] \cdot \nabla T_{1}\left(u_{n}-u_{m}\right) d z \\
& \leq \int_{\Omega_{T}}\left|f_{n}-f_{m}\right| d z+\int_{\Omega}\left|u_{0 n}-u_{0 m}\right| d x=\alpha_{n, m},
\end{aligned}
$$

which implies that

$$
\operatorname{meas}\left(E_{3}\right) \leq \frac{\alpha_{n, m}}{m(h, \delta)} \leq \varepsilon / 3
$$

for all $n, m \geq N_{2}(\varepsilon, \delta)$. It follows from (3.13) and (3.14) that

$$
\operatorname{meas}\left\{(x, t) \in \Omega_{T}:\left|\nabla u_{n}-\nabla u_{m}\right|>\delta\right\} \leq \varepsilon \text { for all } n, m \geq \max \left\{N_{1}, N_{2}\right\},
$$

that is $\left\{\nabla u_{n}\right\}$ is a Cauchy sequence in measure. Then we may choose a subsequence (denote it by the original sequence) such that

$$
\nabla u_{n} \rightarrow v \text { a.e. in } \Omega_{T} \text {. }
$$

Thus, from Proposition 1.6 and $\nabla T_{k}\left(u_{n}\right) \rightarrow \nabla T_{k}(u)$ weakly in $L^{1}\left(\Omega_{T}\right)$, we deduce from Lemma 2.7 that $v$ coincides with the very weak gradient of $u$. Therefore, we have

$$
\nabla u_{n} \rightarrow \nabla u \quad \text { a.e. in } \Omega_{T} \text {. }
$$

Step 3. We shall prove that $u$ is a renormalized solution.

For given $a, k>0$, define the function $T_{k, a}(s)=T_{a}\left(s-T_{k}(s)\right)$ as

$$
T_{k, a}(s)= \begin{cases}s-k \operatorname{sign}(s) & \text { if } k \leq|s|<k+a \\ a \operatorname{sign}(s) & \text { if }|s| \geq k+a \\ 0 & \text { if }|s| \leq k\end{cases}
$$


Using $T_{k, a}\left(u_{n}\right)$ with $u_{n} \geq 0$ as a test function in (3.2), we find

$$
\begin{aligned}
& \int_{\left\{\left|u_{n}\right|>k\right\}} \Theta_{a}\left(u_{n}-k\right)(T) d x-\int_{\left\{\left|u_{0 n}\right|>k\right\}} \Theta_{a}\left(u_{0 n}-k\right) d x \\
& \quad+\int_{\left\{k \leq\left|u_{n}\right| \leq k+a\right\}} D_{\xi} \Phi\left(\nabla u_{n}\right) \cdot \nabla u_{n} d z \leq \int_{\Omega} f_{n} T_{k, a}\left(u_{n}\right) d z
\end{aligned}
$$

which yields that

$$
\int_{\left\{k \leq\left|u_{n}\right| \leq k+a\right\}} D_{\xi} \Phi\left(\nabla u_{n}\right) \cdot \nabla u_{n} d z \leq a\left(\int_{\left\{\left|u_{n}\right|>k\right\}}\left|f_{n}\right| d z+\int_{\left\{\left|u_{0 n}\right|>k\right\}}\left|u_{0 n}\right| d x\right) .
$$

Recalling the fact that $u$ belongs to $C\left([0, T] ; L^{1}(\Omega)\right)$, we have

$$
\lim _{k \rightarrow \infty} \operatorname{meas}\left\{(x, t) \in \Omega_{T}:|u|>k\right\}=0 .
$$

Since $D_{\xi} \Phi\left(\nabla u_{n}\right) \cdot \nabla u_{n} \geq 0$, by Fatou's lemma, (3.11) and (3.16), we get

$$
\begin{aligned}
\int_{\{k \leq|u| \leq k+a\}} D_{\xi} \Phi(\nabla u) \cdot \nabla u d z & =\int_{\Omega_{T}} D_{\xi} \Phi(\nabla u) \cdot \nabla u \chi_{\{k \leq|u| \leq k+a\}} d z \\
& =\int_{\Omega_{T}} \liminf _{n \rightarrow \infty} D_{\xi} \Phi\left(\nabla u_{n}\right) \cdot \nabla u_{n} \chi_{\left\{k \leq\left|u_{n}\right| \leq k+a\right\}} d z \\
& \leq \liminf _{n \rightarrow \infty} \int_{\left\{k \leq\left|u_{n}\right| \leq k+a\right\}} D_{\xi} \Phi\left(\nabla u_{n}\right) \cdot \nabla u_{n} d z
\end{aligned}
$$

Furthermore, since

$$
\begin{aligned}
\int_{\left\{\left|u_{n}\right|>k\right\}}\left|f_{n}\right| d z & =\int_{\left\{\left|u_{n}\right|>k\right\}}\left|f_{n}-f\right| d z+\int_{\left\{\left|u_{n}\right|>k\right\}}|f| d z \\
& \leq \int_{\Omega_{T}}\left|f_{n}-f\right| d z+\int_{\left\{\left|u_{n}\right|>k\right\}}|f| d z,
\end{aligned}
$$

from (3.17) we have

$$
\lim _{k \rightarrow \infty} \lim _{n \rightarrow \infty} \int_{\left\{\left|u_{n}\right|>k\right\}}\left|f_{n}\right| d z=0
$$

Similarly, we know

$$
\lim _{k \rightarrow \infty} \lim _{n \rightarrow \infty} \int_{\left\{\left|u_{0 n}\right|>k\right\}}\left|u_{0 n}\right| d x=0 .
$$

Therefore, passing to the limit first in $n$ then in $k$, we conclude that

$$
\lim _{k \rightarrow \infty} \int_{\left\{(x, t) \in \Omega_{T}: k \leq|u(x, t)| \leq k+a\right\}} D_{\xi} \Phi(\nabla u) \cdot \nabla u d z=0 .
$$

Choosing $a=1$, we obtain the renormalized condition, i.e.,

$$
\lim _{k \rightarrow \infty} \int_{\left\{(x, t) \in \Omega_{T}: k \leq|u(x, t)| \leq k+1\right\}} D_{\xi} \Phi(\nabla u) \cdot \nabla u d z=0 .
$$

Let $S \in C^{\infty}\left(\mathbf{R}^{+}\right)$be such that $\operatorname{supp} S^{\prime} \subset[0, M]$ for some $M>0$. For every nonnegative function $\varphi \in C^{\infty}\left(\bar{\Omega}_{T}\right)$ with $\varphi(x, T)=0, S^{\prime}\left(u_{n}\right) \varphi$ is a test function in 
(3.2). It yields

$$
\begin{aligned}
& \int_{\Omega_{T}} \frac{\partial S\left(u_{n}\right)}{\partial t} \varphi d z+\int_{\Omega_{T}}\left[S^{\prime}\left(u_{n}\right) D_{\xi} \Phi\left(\nabla u_{n}\right) \cdot \nabla \varphi\right. \\
& \left.\quad+S^{\prime \prime}\left(u_{n}\right) D_{\xi} \Phi\left(\nabla u_{n}\right) \cdot \nabla u_{n} \varphi\right] d z=\int_{\Omega_{T}} f_{n} S^{\prime}\left(u_{n}\right) \varphi d z .
\end{aligned}
$$

First we consider the first term on the left-hand side of (3.18). Since $S$ is bounded and continuous, (3.11) implies that $S\left(u_{n}\right)$ converges to $S(u)$ a.e. in $\Omega_{T}$ and weakly-* in $L^{\infty}\left(\Omega_{T}\right)$. Then $\frac{\partial S\left(u_{n}\right)}{\partial t}$ converges to $\frac{\partial S(u)}{\partial t}$ in $D^{\prime}\left(\Omega_{T}\right)$ as $n \rightarrow \infty$, that is

$$
\int_{\Omega_{T}} \frac{\partial S\left(u_{n}\right)}{\partial t} \varphi d z \rightarrow \int_{\Omega_{T}} \frac{\partial S(u)}{\partial t} \varphi d z
$$

For the other terms on the left-hand side of (3.18), because of $\operatorname{supp} S^{\prime} \subset[0, M]$ we know

$$
S^{\prime}\left(u_{n}\right) D_{\xi} \Phi\left(\nabla u_{n}\right)=S^{\prime}\left(u_{n}\right) D_{\xi} \Phi\left(\nabla T_{M}\left(u_{n}\right)\right) .
$$

Using (3.11) and $S \in C^{\infty}\left(\mathbf{R}^{+}\right)$, we have

$$
S^{\prime}\left(u_{n}\right) \rightarrow S^{\prime}(u) \text { a.e. in } \Omega_{T} .
$$

In view of (3.5) and (2.5), we know that

$$
\int_{\Omega_{T}} \Psi\left(D_{\xi} \Phi\left(\nabla T_{M}\left(u_{n}\right)\right)\right) d z \leq C .
$$

Applying Lemma 2.5, Lemma 2.9 and (3.12), we conclude that (up to a subsequence)

$$
D_{\xi} \Phi\left(\nabla T_{M}\left(u_{n}\right)\right) \rightarrow D_{\xi} \Phi\left(\nabla T_{M}(u)\right) \quad \text { weakly in } L^{1}\left(\Omega_{T}\right) .
$$

Then (3.19), (3.20), the boundedness of $S^{\prime}$ and Lemma 2.8 yield that

$$
S^{\prime}\left(u_{n}\right) D_{\xi} \Phi\left(\nabla T_{m}\left(u_{n}\right)\right) \rightarrow S^{\prime}(u) D_{\xi} \Phi\left(\nabla T_{M}(u)\right) \quad \text { weakly in } L^{1}\left(\Omega_{T}\right) .
$$

Noting that

$$
S^{\prime}(u) D_{\xi} \Phi(\nabla u)=S^{\prime}(u) D_{\xi} \Phi\left(\nabla T_{M}(u)\right)
$$

we deduce that

$$
\int_{\Omega_{T}} S^{\prime}\left(u_{n}\right) D_{\xi} \Phi\left(\nabla u_{n}\right) \cdot \nabla \varphi d z \rightarrow \int_{\Omega_{T}} S^{\prime}(u) D_{\xi} \Phi(\nabla u) \cdot \nabla \varphi d z
$$

as $n \rightarrow \infty$.

Moreover, since $S^{\prime \prime}\left(u_{n}\right) \leq 0, \varphi \geq 0$ and

$$
D_{\xi} \Phi\left(\nabla u_{n}\right) \cdot \nabla u_{n} \geq 0
$$

then

$$
-S^{\prime \prime}\left(u_{n}\right) D_{\xi} \Phi\left(\nabla u_{n}\right) \cdot \nabla u_{n} \varphi \geq 0 .
$$

Thus from Fatou's lemma, (3.11) and (3.16), we obtain that

$$
-\int_{\Omega_{T}} S^{\prime \prime}(u) D_{\xi} \Phi(\nabla u) \cdot \nabla u \varphi d z \leq-\liminf _{n \rightarrow \infty} \int_{\Omega_{T}} S^{\prime \prime}\left(u_{n}\right) D_{\xi} \Phi\left(\nabla u_{n}\right) \cdot \nabla u_{n} \varphi d z
$$


Using the strong convergence of $f_{n},(3.11)$ and the Lebesgue dominated convergence theorem, we can pass to the limits as $n \rightarrow \infty$ for the right-hand side of (3.18) to conclude that

$$
\begin{aligned}
& -\int_{\Omega} S\left(u_{0}\right) \varphi(x, 0) d x-\int_{0}^{T} \int_{\Omega} S(u) \frac{\partial \varphi}{\partial t} d z \\
& \quad+\int_{0}^{T} \int_{\Omega}\left[S^{\prime}(u) D_{\xi} \Phi(\nabla u) \cdot \nabla \varphi+S^{\prime \prime}(u) D_{\xi} \Phi(\nabla u) \cdot \nabla u \varphi\right] d z \\
& \geq \int_{0}^{T} \int_{\Omega} f S^{\prime}(u) \varphi d z
\end{aligned}
$$

for all $k>0$ and $\varphi \in C^{1}\left(\bar{\Omega}_{T}\right)$ with $\varphi \geq 0$ and $\left.\varphi\right|_{\Sigma}=0$. This finishes the proof.

Acknowledgements. The authors wish to thank the anonymous referee for careful reading of the early version of this manuscript and bringing several reference papers to their attention and providing many valuable suggestions and comments. The first author was supported by Hei Long Jiang Postdoctoral Foundation and the Natural Scientific Research Innovation Foundation in Harbin Institute of Technology. The second author was supported in part by the NSFC under Grant 10990013.

\section{References}

[1] ACERBI, E., and N. Fusco: A regularity theorem for minimizers of quasi-convex integrals. Arch. Ration. Mech. Anal. 99, 1987, 261-281.

[2] Bénilan, P., L. Boccardo, T. Gallouët, R. Gariepy, M. Pierre, and J. L. Vazquez: An $L^{1}$-theory of existence and uniqueness of solutions of nonlinear elliptic equations. - Ann. Sc. Norm. Super. Pisa Cl. Sci. (4) 22, 1995, 241-273.

[3] Blanchard, D., and F. Murat: Renormalised solutions of nonlinear parabolic problems with $L^{1}$ data: Existence and uniqueness. - Proc. Roy. Soc. Edinburgh Sect. A 127:6, 1997, 1137-1152.

[4] Blanchard, D., F. Murat, and H. Redwane: Existence and uniqueness of a renormalized solution for a fairly general class of nonlinear parabolic problems. - J. Differential Equations $177: 2,2001,331-374$.

[5] Blanchard, D., and H. Redwane: Renormalized solutions for a class of nonlinear evolution problems. - J. Math. Pures Appl. (9) 77, 1998, 117-151.

[6] Boccardo, L., A. Dall'Aglio, T. Gallouët, and L. Orsina: Nonlinear parabolic equations with measure data. - J. Funct. Anal. 147:1, 1997, 237-258.

[7] Bocchrdo, L., and T. GallouËт: Nonlinear elliptic and parabolic equations involving measure data. - J. Funct. Anal. 87:1 ,1989, 149-169.

[8] Boccardo. L., and T. GallouËt: Nonlinear elliptic equations with right-hand side measures. - Comm. Partial Differential Equations 17:3-4, 1992, 641-655.

[9] Boccardo, L., D. Giachetti, J. I. Diaz, and F. Murat: Existence and regularity of renormalized solutions for some elliptic problems involving derivations of nonlinear terms. - J. Differential Equations 106, 1993, 215-237.

[10] CAI, Y., and S. Zhou: Existence and uniqueness of weak solutions for a non-uniformly parabolic equation. - J. Funct. Anal. 257, 2009, 3021-3042.

[11] Cellina, A.: On the validity of the Euler-Lagrange equation. - J. Differential Equations 171, 2001, 430-442.

[12] Chapman, S., and T. G. Cowling: Mathematical theory of nonuniform gases. - Cambridge Univ. Press, 3rd edition, 1990. 
[13] Chen, Y.: Parabolic partial differential equations of second order. - Peking Univ. Press, Beijing, 2003 (in Chinese).

[14] Dacorogna, B.: Direct methods in the caculus of variations. - Springer-Verlg, BerlinHeidelberg, 1989.

[15] Dal Maso, G., F. Murat, L. Orsina, and A. Prignet: Renormalized solutions of elliptic equations with general measure data. - Ann. Sc. Norm. Super. Pisa Cl. Sci. (4) 28:4, 1999, $741-808$.

[16] Dall'Aglio, A.: Approximated solutions of equations with $L^{1}$ data. Application to the $H$ convergence of quasi-linear parabolic equations. - Ann. Mat. Pura Appl. (4) 170, 1996, 207-240.

[17] Duc, D. M., and J. EELls: Regularity of exponetional harmonic functions. - Internat. J. Math. 2, 1991, 395-408.

[18] DiBenedetto, E.: Degenerate parabolic equations. - Springer-Verlag, Berlin, 1993.

[19] DiBenedetto, E.: Real analysis. - Birkhäuser, Boston, 2002.

[20] DiPerna, R. J., and P.-L. Lions: On the Cauchy problem for Bolzmann equations: global existence and weak stability. - Ann. of Math. (2) 130:2, 1989, 321-336.

[21] Evans, L. C.: Partial differential equations. - Amer. Math. Soc., Providence, Rhode Island, 1998.

[22] Fuchs, M., and G. Mingione: Full $C^{1, \alpha}$-regularity for free and contrained local minimizers of elliptic variational integrals with nearly linear growth. - Manuscripta Math. 102:2, 2000, $227-250$.

[23] Hiriart-Urruty, J.-B., and C. Lemaréchal: Fundamentals of convex analysis. - SpringerVerlag, Berlin-Heidelberg, 2001.

[24] Lieberman, G. M.: On the regularity of the minimizer of a functional with exponetial growth. - Comment. Math. Univ. Carolin. 33:1, 1992, 45-49.

[25] Lions, J.-L.: Quelques méthodes de résolution des problèmes aux limites non linéaire. - Dunod et Gauthier-Villars, Paris, 1969.

[26] Lions, P.-L.: Mathematical topics in fluid mechanics, Vol. 1: Incompressible models. - Oxford Univ. Press, Oxford, 1996.

[27] Lions, P.-L., and F. Murat: Solutions renormalisées d'équations elliptiques non linéaires. Unpublished.

[28] Naito, H.: On a local Hölder continuity for a minimizer of the exponential energy functinal. - Nagoya Math. J. 129, 1993, 97-113.

[29] Prignet, A.: Existence and uniqueness of "entropy" solutions of parabolic problems with $L^{1}$ data. - Nonlinear Anal. 28:12, 1997, 1943-1954.

[30] Royden, H. L.: Real analysis. - Macmillan Company, New York, 2nd edition, 1968.

[31] SaAdoune, M., and M. Valadier: Extraction of a "good" subsequence from a bounded sequence of integrable functions. - J. Convex Anal. 2, 1995, 345-357.

[32] Serrin, J.: Pathological solutions of elliptic differential equations. - Ann. Sc. Norm. Super. Pisa Cl. Sci. (3) 18, 1964, 385-387.

[33] Zhang, C., and S. Zhou: Renormalized and entropy solutions for nonlinear parabolic equations with variable exponents and $L^{1}$ data. - J. Differential Equations 248:6, 2010, 1376-1400.

[34] ZhANG, C., and S. Zhou: Entropy solutions for a non-uniformly parabolic equation. Manuscripta Math. 131:3-4, 2010, 335-354.

Received 18 March 2011

Revised received 18 July 2011 